\title{
Reduction of microtrenching and island formation in oxide plasma etching by employing electron beam charge neutralization
}

\author{
M. Watanabe, ${ }^{\text {a) }}$ D. M. Shaw, and G. J. Collins \\ Department of Electrical Engineering, Colorado State University, Fort Collins, Colorado 80523
}

(Received 23 April 2001; accepted for publication 13 August 2001)

\begin{abstract}
During plasma etching of oxide thin-film patterns, nonuniform charge buildup within etching features results in formation of microtrenches. Near the etch endpoint, the underlying film layer adjacent to the feature edges is exposed first, leaving an oxide island in the feature center and potentially causing underlayer damage before the endpoint is reached. Herein, a directional electron flux is added to the plasma ion flux incident on the etching substrate with the goal of minimizing microtrenching and oxide island formation. Scanning electron microscopic images of patterns etched with added electron irradiation show a reduction in microtrenching and oxide island formation as compared to patterns etched under identical conditions without electron irradiation. A computer simulation shows that the added electron irradiation reduces microtrenching by allowing more uniform ion flux to reach the feature bottom. (c) 2001 American Institute of Physics.
\end{abstract}

[DOI: $10.1063 / 1.1413726]$

In plasma pattern etching, positive ions accelerated to the substrate by the rf biased electrode sheath provide directionality for forming vertical feature profiles. Simultaneously, plasma electrons, due to their random thermal motion, tend to impinge on the sidewalls near the top of the feature. Over the course of the etch process, the feature bottom charges positively and the sidewalls charge negatively, creating undesired local electric fields within the feature. Subsequent ion trajectories tend to be deflected towards the sidewalls, resulting in an increased etch rate near the edges of feature bottom and decreased etch rate at the center. As device feature sizes continue to decrease, the local electric field is becoming problematic by causing nonideal etch profiles, such as microtrenching in oxide ${ }^{1,2}$ and notching in polysilicon. ${ }^{3-7}$ Recent theoretical work has even suggested that feature charging may lead to complete etch stoppage in high aspect ratio contact holes. ${ }^{8}$

Figure 1 schematically illustrates an oxide trench profile near the etch endpoint. Microtrenching has exposed the underlying silicon substrate near the bottom edges, leaving an oxide island in the center. Since the conducting silicon underlayer does not allow positive charge accumulation, ions tend to be further deflected towards the feature edges, making the clearing of the oxide island difficult. Frequently, when the etch time is extended in an attempt to remove the oxide islands (termed "overetching"), microtrenches are inadvertently transferred into the underlying silicon, causing device damage. In extreme cases, the oxide island remains even after overetching, causing high electrical contact resistance to the underlayer in the completed device. Therefore, a charge neutralization method to reduce microtrenching and oxide island formation within etching features is desirable.

Energetic electron flux from a hot tungsten filament incident on the etching substrate has been proposed to reduce notching during poly-silicon etching and reactive ion etching

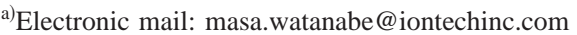

lag during oxide etching. ${ }^{9}$ Herein, we investigate a cold cathode electron irradiation technique to reduce microtrenching and island formation during oxide etching. Figure 2 schematically shows the addition of electron irradiation during oxide etching for charge neutralization. Energetic electron flux normal to the wafer surface reaches the feature bottom and neutralizes the positive charge on the oxide island. The local electric field within the feature is reduced, and straighter ion trajectories are obtained. Therefore, more ideal etching profiles and reduced overetch time can be expected.

Figure 3 shows a schematic drawing of the inductively coupled plasma (ICP) etching system used herein, with the electron beam source located $14 \mathrm{~cm}$ away from the wafer electrode. We employ a cold cathode secondary electron beam source ${ }^{10,11}$ that is compatible with reactive gases. The source produces electron current densities of $\sim 1 \mathrm{~mA} / \mathrm{cm}^{2}$ at the wafer surface. Electron beam energy is set to $850 \mathrm{eV}$, and the wafer sheath $\mathrm{rf}(13.56 \mathrm{MHz})$ potential is set to $200 \mathrm{~V}_{p-p}$. Plasma feedstock gas is 6 mTorr of $\mathrm{CF}_{4}: \mathrm{H}_{2}(10: 1)$.

Oxide films on silicon substrates with $0.45 \mu \mathrm{m}$ diameter hole patterns defined by photoresist masks are placed on the

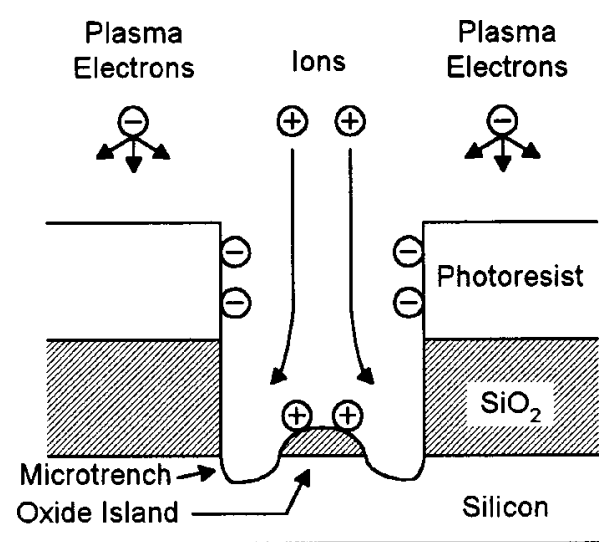

FIG. 1. Schematic illustration of microtrench and oxide island formation near the etch endpoint in oxide feature etching. 


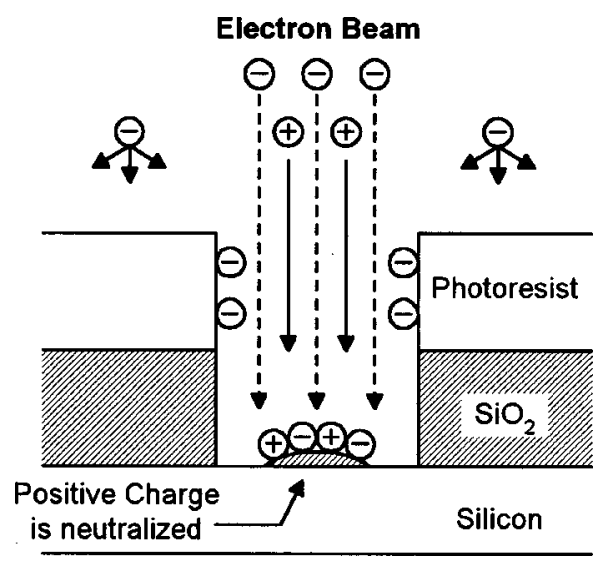

FIG. 2. Electron beam irradiation during etching neutralizes positive charge buildup on the oxide island and reduces the local electric field inside the etching feature. The result is a more uniform ion flux distribution along the feature bottom, increasing etch rate uniformity, and reducing microtrenching.

rf biased wafer electrode. Substrates are etched under identical plasma conditions both with and without electron beam irradiation. Figure 4 illustrates the effects of the electron beam neutralization within the etching feature via scanning electron microscopic (SEM) imaging. Figure 4(a) shows an oxide contact hole near the endpoint etched without electron beam irradiation. An oxide island and two deep microtrenches are clearly visible. The flatter bottom corners of the contact hole etched with simultaneous electron beam irradiation in Fig. 4(b) clearly shows reduced microtrench and oxide island formation.

A simplified two-dimensional computer simulation, based on the previous work of Arnold and Sawin, ${ }^{1}$ is used to verify that the observed microtrench and oxide island reduction is due to charge neutralization by the electron beam irradiation. In the simulation, mono-energetic ions with energy $E_{i}=100 \mathrm{eV}$, thermal electrons with electron temperature $T_{e}=4 \mathrm{eV}$, and beam electrons with energy $E_{e}=850 \mathrm{eV}$ are incident on a trench-shaped oxide feature with zero initial charge. The local electric field created as the charge builds up is computed, and subsequent charged particle trajectories are deflected. Steady-state conditions are obtained when the

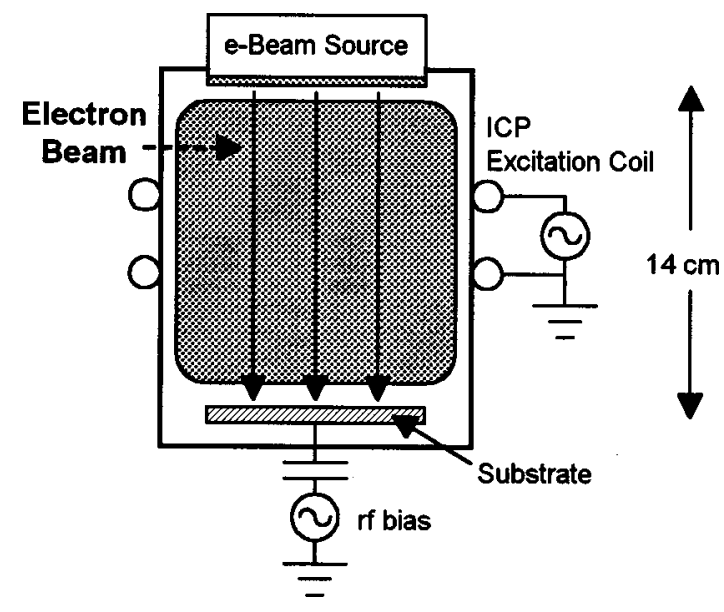

FIG. 3. Schematic of the ICP etch system including the cold cathode electron beam source. Electrons from the cold cathode beam source propagate through the plasma and impinge on the etching substrate simultaneously with plasma ions. (a)

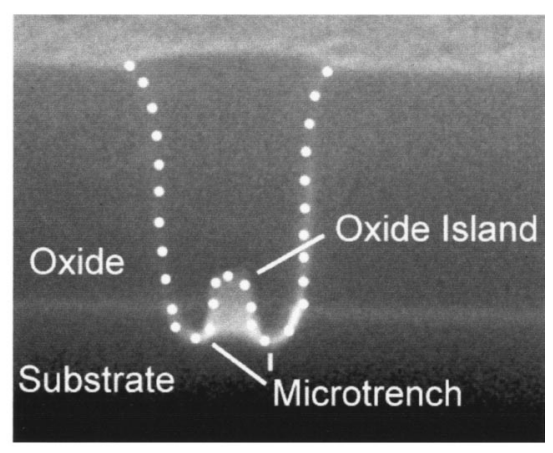

(b)

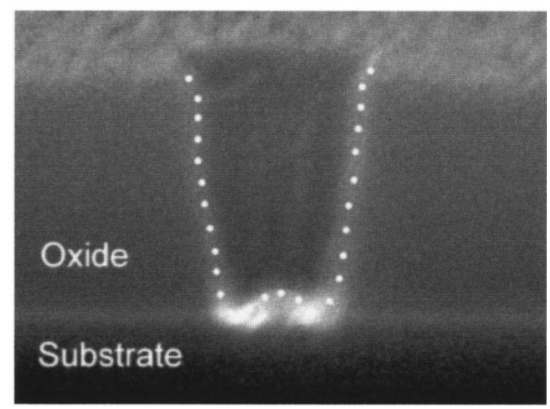

FIG. 4. SEM images of $0.45 \mu \mathrm{m}$ oxide contact holes near the etch endpoint. The feature edges are highlighted with a dotted line as a guide for the eye due to the difficulty in reproducing the SEM images. (a) Microtrenching and oxide island formation on the contact hole bottom are evident without electron beam irradiation. (b) Although still present, island formation and microtrenching are much reduced by simultaneous electron beam irradiation.

net charge flux to any dielectric surface becomes zero. In order to simulate the conducting substrate exposed near the etch endpoint, the edges of the feature bottom are not allowed to charge up, while the center of the feature bottom can accumulate charge to replicate the insulating oxide island. The oxide is considered a perfect insulating surface. Any breakdown of the oxide or leakage current to the substrate is neglected. The simulation is run both with and without the electron beam to study its effect on the ion trajectories.

The results of the simulation are shown in Fig. 5. The solid line shows nonuniform ion flux distribution along the feature bottom when only ion and low-energy plasma electron fluxes are considered. Incident ions are deflected towards the sidewalls by the electric field created primarily between the positively charged oxide island and the conducting substrate where charge accumulation does not occur. The nonuniform ion flux distribution results in an increased etch rate at the feature bottom edges and reduced etch rate at the center, enhancing microtrenching and oxide island formation.

When simultaneous electron beam irradiation is added to the ion and low-energy plasma electron fluxes, the calculated ion flux distribution becomes more uniform as shown with the dashed line in Fig. 5. Here, positive charge neutralization by the energetic beam electrons incident on the oxide island reduces the local electric field inside the feature. The resulting ion trajectories are straighter, resulting in more uniform 


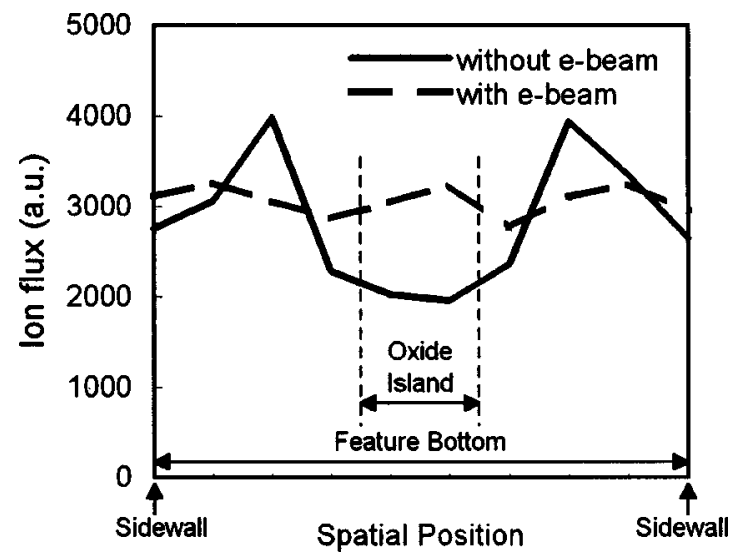

FIG. 5. Simulated ion flux distribution reaching the oxide feature bottom near the etch endpoint with and without simultaneous directional electron beam irradiation. With no electron beam flux, the local electric field inside the etching feature deflects ion trajectories towards the edges. The local electric field is reduced due to charge neutralization on the oxide island with electron beam irradiation, resulting in a more uniform ion flux distribution.

ion flux and implying a spatially uniform etch rate along the feature bottom. The results of our simple particle trajectory simulation agree well with the experimental etch profiles shown in the SEM images of Fig. 4.

In addition to the reduced ion flux uniformity, in the case without electron beam irradiation, the simulation also indicates that the energy of the ions incident on the oxide island is reduced by the decelerating effect of the unintentional electric field. However, the ions incident on the conducting feature bottom edges maintain their initial energy. By reducing the unintentional electric field with electron beam irradiation, a more uniform ion energy distribution along the feature bottom is obtained. Although the ion flux distribution strongly determines the etch rate along the feature bottom, the incident ion energy distribution could also influence the local etch rate. We are currently studying the combined effects of both spatial ion flux variation and ion energy variations on the local etch rates.

In summary, we have explored charge neutralization using electron beam irradiation to reduce microtrench and oxide island formation during conventional plasma etching of oxide patterns. SEM images indicate that the technique results in a dramatic reduction of microtrench and oxide island formation near the etch endpoint. A computer simulation of ion trajectories within the etching feature verifies that the charge neutralization reduces the unintentional electric field responsible for microtrench/oxide island formation.

Helpful discussions with Dr. J. Meyer of Agilent Technologies are gratefully acknowledged. This work was supported by the National Science Foundation (ECS-0097061).

${ }^{1}$ J. C. Arnold and H. H. Sawin, J. Appl. Phys. 70, 5314 (1991).

${ }^{2}$ M. Schaepkens and G. S. Oehrlein, Appl. Phys. Lett. 72, 1293 (1998).

${ }^{3}$ T. Nozawa, T. Kinoshita, T. Nishizuka, A. Narai, T. Inoue, and A. Nakaue, Jpn. J. Appl. Phys., Part 1 34, 2107 (1995).

${ }^{4}$ T. Kinoshita, M. Hane, and J. P. McVittie, J. Vac. Sci. Technol. B 14, 560 (1996)

${ }^{5}$ N. Fujiwara, S. Ogino, T. Maruyama, and M. Yoneda, Plasma Sources Sci. Technol. 5, 126 (1996).

${ }^{6}$ N. Fujiwara, T. Maruyama, and M. Yoneda, Jpn. J. Appl. Phys., Part 1 35, 2450 (1996).

${ }^{7}$ T. H. Ahn, K. Nakamura, and H. Sugai, Plasma Sources Sci. Technol. 5, 139 (1996).

${ }^{8}$ J. Matsui, N. Nakano, Z. L. Petrovic, and T. Makabe, Appl. Phys. Lett. 78, 883 (2001).

${ }^{9}$ A. K. Quick, Ph.D. thesis, University of Wisconsin-Madision, 1998.

${ }^{10}$ D. M. Shaw, M. Watanabe, H. Uchiyama, and G. J. Collins, Appl. Phys. Lett. 75, 34 (1999).

${ }^{11}$ D. M. Shaw, M. Watanabe, G. J. Collins, and H. Sugai, Jpn. J. Appl. Phys., Part 1 38, 4590 (1999). 